\title{
Numerical simulations of the Richtmyer-Meshkov instability in solid-vacuum interfaces using calibrated plasticity laws
}

\author{
A. López Ortega, M. Lombardini, D. I. Pullin, and D. I. Meiron \\ Graduate Aerospace Laboratories, California Institute of Technology, Pasadena, California 91125, USA
}

(Received 4 October 2013; published 28 March 2014)

\begin{abstract}
The Richtmyer-Meshkov instability of interfaces separating elastic-plastic materials from vacuum (heavy-light configuration) is studied by means of computational techniques. A fully Eulerian multimaterial algorithm that solves consistently the Euler equations and the time evolution of the deformations in the material is applied to three distinct materials (copper, aluminum, and stainless steel). If a perfectly plastic constitutive relation is considered, an empirical law is computed that relates the long-term perturbation amplitude of the interface, its maximum growth rate, the initial density, and the yield stress of the material. It is shown that this linear relation can be extended to materials that follow more complex plastic behavior which can account for rate dependency, hardening, and thermal softening, and to situations in which small-perturbation theory is no longer valid. In effect, the yield stress computed from measurements of the long-term amplitude and maximum growth rate closely matches the von Mises stress found at the interface of solid materials for a wide range of cases with different initial parameters.
\end{abstract}

DOI: 10.1103/PhysRevE.89.033018

PACS number(s): 47.40.Nm, 62.50.Ef, 52.50.Lp

\section{INTRODUCTION}

The Richtmyer-Meshkov instability [1,2] describes the behavior of a perturbed interface separating two materials of different densities when processed by a shock wave. The misalignment between the density and pressure gradients, which are perpendicular to the interface and the shock wave, respectively, produces the deposition of vorticity at the interface which leads to unbounded growth of the interface in most cases. This instability can also occur in free surfaces separating continuous media from vacuum since the baroclinic term in the vorticity equation does not vanish. The free-surface Richtmyer-Meshkov instability can be regarded as the limiting case of a free-slip interface separating a solid or fluid material from a very rarified gas and it is shown that a vortex sheet can be mathematically defined in this situation [3]. The Richtmyer-Meshkov instability has been extensively studied from the fluid dynamics perspective and appears in problems of interest in magnetohydrodynamics [4], plasma physics [5], astrophysics [6-9], and solid mechanics. In this last field, multiple analytical and numerical approaches based on the linearization of the equations of motion [10-12] have been proposed to describe the time evolution of the interface when the two materials separated by the interface are purely elastic. The results show that the interface is always stable in this configuration, owing to the shear waves present in solid materials and their capability to transport the initially deposited vorticity away from the interface and into the materials. The analytical expressions derived in [12] show that a sufficient condition for interface stability is that one of the materials needs to be purely elastic and have finite shear strength, while the other material can be a fluid or even be substituted with vacuum. Purely analytical models are not readily available when elastic-plastic solid materials are considered, as plasticity theory is intrinsically nonlinear, with yield criteria being usually based on tensor norms. Thus, the study of the Richmyer-Meshkov instability for elastic-plastic solids must rely on approaches that employ numerical techniques to some extent.
The first attempt to characterize this flow was due to Piriz et al. [13], who considered a light-heavy solid-vacuum configuration (i.e., a shock wave initiating at the interface and traveling into the solid) and a sinusoidally perturbed interface. They constructed an analytical model for small perturbations of the interface that relied on numerical simulations using the finite-element code ABAQUS for parameter fitting. This model suggested that the behavior of the interface has three distinct phases. In the first one, the solid enters the plastic state after being processed by the shock, and the interface increases its perturbation amplitude at a constant growth rate. In the second phase, relaxation of stresses occurs and the growth rate decreases drastically as the solid adjacent to the interface leaves the plastic state. Finally, in the long-term behavior, the interface oscillates around an average amplitude in a fashion that mimics the behavior of the interface for elastic materials. The relationship for the long-term amplitude of the interface as a function of the material parameters and initial conditions reads

$$
\bar{\eta}=\eta_{0}+C \frac{\rho_{0} \dot{\eta}^{2}}{k \sigma_{Y}},
$$

where $\bar{\eta}$ and $\eta_{0}$ are the long-term and initial interface amplitudes, respectively, $\dot{\eta}$ is the initial growth rate (which follows the classical Richtmyer result $\dot{\eta}=\eta_{0} k \Delta V_{i}$, where $\Delta V_{i}$ is the change in the interface mean velocity due to the interaction with the shock), $\rho_{0}$ is the unstressed density of the solid, $k$ is the wave number of the interface perturbations, and $\sigma_{Y}$ is the yield stress in a perfectly plastic inelastic model. The value of the coefficient $C$ was determined to be $\approx 0.29$, irrespective of the material (as long as perfect plasticity is considered).

Dimonte et al. [14] later considered the solid-vacuum heavy-light configuration (i.e., a shock wave initiated in the solid which reflects as an expansion upon reaching the interface) by means of computational techniques and experiments. The hydrocode PAGOSA [15] was employed to run a series of simulations modeling perfectly plastic copper and to establish that under certain initial conditions, the interface can grow unstably in time, exceeding the range of validity of the 


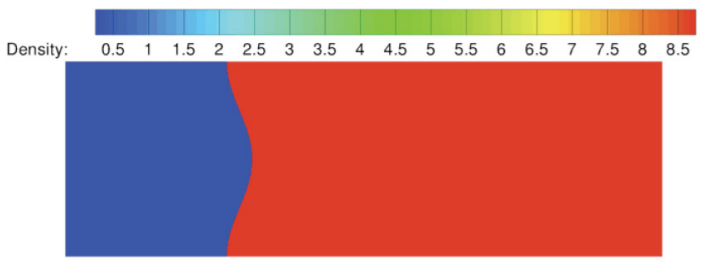

(a)Initial condition

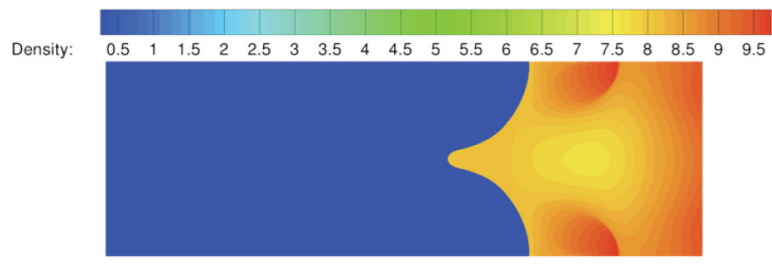

(c) $t V_{b u} k=1.15$

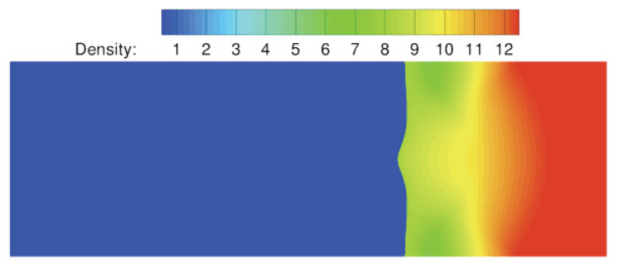

(b) $t V_{b u} k=-0.15$

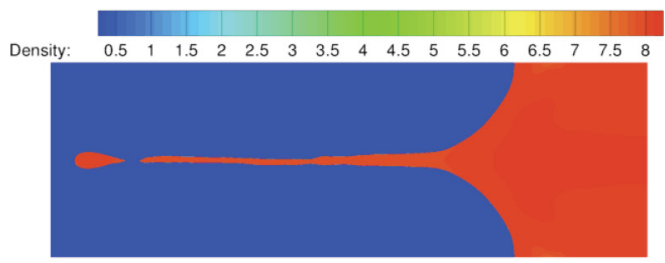

(d) $t V_{b u} k=9.55$

FIG. 1. (Color online) Sequence of density contour plots that describe the evolution of the Richtmyer-Meshkov instability of a planarvacuum interface. In this particular case copper is used with parameters $\sigma_{Y}=0.5 \mathrm{GPa}, \eta_{0} k=0.4$, and $M_{s}=1.67$. The interface exhibits unstable behavior in all phases which results in the formation of ejecta.

hypotheses in [13]. This unbounded growth is associated with high initial amplitudes, low yield stresses, and large shock strength. The law allowing determination of the maximum amplitude as a function of the initial parameters and material properties was modified to

$$
\bar{\eta}_{s p}=C \frac{\rho_{0} \dot{\eta}_{s p, \max }^{2}}{k \sigma_{Y}},
$$

where the subscript $s p$ denotes the amplitude of the "spikes." In numerical simulations, the linear regime in which the perturbations follow a sinusoidal shape is soon abandoned. The regions of the solid material that penetrate into the initial vacuum region tend to become narrow, forming spikes, while the complementary regions acquire the shape of "bubbles." There are multiple reasons for modifying the expression proposed in [13]. In the first place, in heavy-light configurations, the interface undergoes what is called a "phase reversal" due to an initial growth rate that is negative. For that reason, in this expression, the authors considered the phase reversal (i.e., the time at which the amplitude of the interface passes through zero) as the starting point for measuring perturbations. In addition, in numerical simulations of the nonlinear state, the growth rate does not reach immediately its maximum value and, because of this, the maximum value $\dot{\eta}_{s p}^{\max }$ is computed and used in the expression. Dimonte et al.'s result for the coefficient was $C=0.24$ and it is argued that the lower value with respect to that computed in [13] is due to the phase-reversal process. Figure 1 depicts a sequence of density contour plots for a case (perfectly plastic copper with yield stress $\sigma_{Y}=0.5 \mathrm{GPa}$, initial amplitude $\eta_{0} k=0.4$, and shock Mach number $M_{s}=1.67$ ) for which the interface grows unstably and ultimately ejecta are formed. Time is set to zero at the completion of the phase reversal and is nondimensionalized with the wave number and the maximum growth rate of the bubbles, following the procedure employed in [14]. Figure 1(a) shows the initial single-mode perturbation of the interface. In Fig. 1(b) the shock has already processed the interface and the phase reversal is in progress (due to the large initial perturbation, this process in nonlinear). The interface transitions to a shape consisting of thin spikes and complementary bubbles [Fig. 1(c)]. Finally, in Fig. 1(d) the tip of the bubble detaches, forming ejecta. The latter process is an effect of the way our algorithm is implemented (i.e., no fracture algorithm was implemented). However, experiments reported in [14] seem to indicate that formation of ejecta indeed occurs.

In this article, we report results that first use a multimaterial solid mechanics algorithm based on an Eulerian framework to run simulations of the solid-vacuum Richtmyer-Meshkov instability and independently estimate a value of the coefficient $C$. For this purpose, we run a series of simulations following Dimonte et al.'s problem configuration but employ three different materials: copper, aluminum, and stainless steel. Perfect plasticity behavior is assumed in this section. The initial conditions of the interface, shock Mach numbers, and yield stress are varied in order to construct a linear regression from which $C$ can be determined. The numerical implementation and equations are briefly described in Sec. II, while the results of the linear regression are exposed in Sec. III. Finally, we employ calibrated plasticity laws for the same three materials named above in order to show that an "effective" yield stress can be determined by applying Eq. (2) to the results of the simulation and that this value is very close to the maximum von Mises stress measured for the solid material at the interface.

\section{EQUATIONS AND NUMERICAL IMPLEMENTATION}

In a Eulerian frame of reference $\left\{x_{1}, x_{2}, x_{3}\right\}$, the equations that describe the motion of elastic-plastic solid media are

$$
\begin{aligned}
\frac{\partial \rho}{\partial t}+\frac{\partial \rho u_{i}}{\partial x_{i}} & =0 \\
\frac{\partial \rho u_{i}}{\partial t}+\frac{\partial\left(\rho u_{i} u_{j}-\sigma_{i j}\right)}{\partial x_{j}} & =0
\end{aligned}
$$




$$
\begin{aligned}
& \frac{\partial \rho\left(e+u_{i} u_{i} / 2\right)}{\partial t}+\frac{\partial\left[\rho\left(e+u_{i} u_{i} / 2\right) u_{j}-\sigma_{i j} u_{i}\right]}{\partial x_{j}}=0, \\
& \frac{\partial g_{i j}^{e}}{\partial t}+\frac{\partial\left(g_{i k}^{e} u_{k}\right)}{\partial x_{j}}=u_{k}\left(\frac{\partial g_{i k}^{e}}{\partial x_{j}}-\frac{\partial g_{i j}^{e}}{\partial x_{k}}\right)+L_{i k}^{p} g_{k j}^{e},
\end{aligned}
$$

where $\rho, \mathbf{u} \equiv \partial \mathbf{x} / \partial t, \sigma$, and $e$ are the density, velocity, Cauchy stress, and specific internal energy, respectively. The deformation history in the solid is tracked by the so-called elastic inverse deformation tensor $\mathbf{g}^{e}$. The tensor $\mathbf{L}^{p}$ reflects the influence of the plasticity model on the elastic deformations and is modeled following a Maxwell relaxation,

$$
\mathbf{L}^{p}=\frac{1}{2 \mu \tau} \mathbf{g}^{e} \boldsymbol{\sigma}^{\prime} \mathbf{g}^{e-1}
$$

Here $\sigma^{\prime}=\sigma-\operatorname{tr}(\sigma) / 3$ is the deviatoric Cauchy stress, $\mu$ is the shear modulus, and $\tau$ is the relaxation time. A closure relation is provided by means of a hyperelastic constitutive law [16] $e=e\left(\mathbf{g}^{e}, \varsigma\right)[16]$ with $\varsigma$ being the entropy, such that

$$
\sigma_{i j}=-\rho g_{k i}^{e} \frac{\partial e}{\partial g_{k j}^{e}} .
$$

The Godunov-Romenski hyperelastic constitutive law $[17,18]$ is employed for the modeling of elastic-plastic solids. This closure relation considers contributions to the internal energy related to shear and hydrostatic deformations, respectively:

$$
\begin{aligned}
e & =e_{s}+e_{h}, \\
e_{s} & =2 c_{s}(\rho)^{2} I^{2}, \quad I^{2}=\frac{\left(I_{1}^{\mathbf{H}^{e}}\right)^{2}}{3}-I_{2}^{\mathbf{H}^{e}}, \\
e_{h} & =\frac{K}{2 \alpha^{2}}\left[\left(\frac{\rho}{\rho_{0}}\right)^{\alpha}-1\right]^{2}+c_{v} T_{0}\left(\frac{\rho}{\rho_{0}}\right)^{\gamma}\left[\exp \left(\frac{\varsigma}{c_{v}}\right)-1\right],
\end{aligned}
$$

where $c_{s}(\rho)=\mu / \rho=\mu_{0} \rho^{\beta} / \rho_{0}^{\beta+1}$, and $I_{1}^{\mathbf{H}^{e}}$ and $I_{2}^{\mathbf{H}^{e}}$ are the first two invariants of the elastic Hencky strain tensor $\mathbf{H}^{e}=$ $\frac{1}{2} \ln \left(\mathbf{g}^{e-1} \mathbf{g}^{e-T}\right)$ (i.e., $I_{1}^{\mathbf{H}^{e}}=\operatorname{tr}\left(\mathbf{H}^{e}\right)$ and $I_{2}^{\mathbf{H}^{e}}=\left\{\left[\operatorname{tr}\left(\mathbf{H}^{e}\right)\right]^{2}-\right.$ $\left.\left.\operatorname{tr}\left(\mathbf{H}^{e 2}\right)\right\} / 2\right)$. Material parameters for the materials considered in this study (OFHC copper, aluminum Al 6061-T6, and stainless steel SS 304) are listed in Table I.

TABLE I. Parameters for OFHC copper, aluminum Al 6061-T6, and stainless steel SS 304.

\begin{tabular}{lcccc}
\hline \hline Parameter & $\mathrm{Cu}$ & $\mathrm{Al}$ & $\mathrm{SS}$ & Units \\
\hline$\rho_{0}$ & 8.930 & 2.703 & 7.903 & $\mathrm{~g} \mathrm{~cm}^{-3}$ \\
$K$ & $15.28 \times 10^{6}$ & $28.23 \times 10^{6}$ & $21.03 \times 10^{6}$ & $\mathrm{~m}^{2} \mathrm{~s}^{-2}$ \\
$c_{v}$ & $3.9 \times 10^{2}$ & $9 \times 10^{2}$ & $4.55 \times 10^{2}$ & $\mathrm{~J} \mathrm{~kg}^{-1} \mathrm{~K}^{-1}$ \\
$T_{0}$ & 298 & 298 & 298 & $\mathrm{~K}$ \\
$\mu_{0}$ & 39.38 & 26.36 & 77.6 & $\mathrm{GPa}$ \\
$\alpha$ & 1 & 0.627 & 0.815 & \\
$\beta$ & 3 & 2.288 & 2.710 & \\
$\gamma$ & 2 & 1.484 & 1.697 & \\
\hline \hline
\end{tabular}

The inelastic behavior depends on the relaxation time parameter $\tau$, whose general expression takes the form

$$
\begin{aligned}
\tau & =\tau_{0}\left(N_{0}+M \epsilon\right)^{-1} \exp \left[\frac{\sigma_{0} f_{H}\left(\sigma_{m}, \epsilon\right) f_{T}(\rho, \varsigma)}{\sigma_{m}}\right], \\
\sigma_{m} & :=\sqrt{\frac{3}{2}}\left\|\sigma^{\prime}\right\|, \quad \epsilon:=\left(C_{H} \frac{\varsigma^{P}}{c_{v} \sigma_{m}}\right)
\end{aligned}
$$

for copper, and

$$
\tau=\tau_{0}^{\prime}\left(\frac{\sigma_{0} f_{H}\left(\sigma_{m}, \epsilon\right) f_{T}(\rho, \varsigma)}{\sigma_{m}}\right)^{n}
$$

for aluminum and stainless steel. In the above expressions, $\tau_{0}$ is a reference relaxation time, $N_{0}$ is the initial dislocation density, $M$ a multiplication parameter, $D$ is the characteristic drag stress, and $C_{H}$ is a material parameter. $\varsigma^{P}$ is the contribution to entropy that is attributed to previous plastic work and needs to be tracked as an additional variable in the system of equations. The functions $f_{H}$ and $f_{T}$ represent the response to nonlinear strain hardening and thermal softening, respectively, and take the forms

$$
\begin{aligned}
f_{H}\left(\sigma_{m}, \epsilon\right) & =1+H \epsilon^{n_{1}}, \\
f_{T}(\rho, \varsigma) & =\left(1-\left\langle\frac{T(\rho, \varsigma)-T_{0}}{T_{M}(\rho)-T_{0}}\right\rangle\right)^{m},
\end{aligned}
$$

with $\langle\phi\rangle=(\phi+|\phi|) / 2$ and where $H$ and $n_{1}$ are material parameters, $T_{M}=T_{M_{0}}\left(\rho / \rho_{0}\right)^{2\left(\gamma_{s}-1\right)-2 / 3}$ is the melting temperature found from the Lindemann theory, and the temperature $T$ is obtained from differentiation of the internal energy expression (6) with respect to the entropy (i.e., $T=\partial e /\left.\partial \varsigma\right|_{\mathbf{g}^{c}}$ ). The values for the parameters shown in Table II have been calibrated to fit with minimum error the experimental data from tensile tests [19] (aluminum 6061-T6 and stainless steel $304)$ and from [20] (OFHC copper).

A detailed description of the numerical discretization of the equations of motion and the computation of the plasticity models can be found in [21-23]. As a summary, the equations of motion for elastic-plastic solids (3) are implemented in the AMROC framework [24], a parallel implementation of the adaptive mesh refinement algorithm of Berger and Collela [25] for solving generic systems of hyperbolic partial differential

TABLE II. Parameters in plasticity model for OFHC copper, aluminum Al 6061-T6, and stainless steel SS 304.

\begin{tabular}{lcccc}
\hline \hline Parameter & $\mathrm{Cu}$ & $\mathrm{Al}$ & $\mathrm{SS}$ & Units \\
\hline$\sigma_{0}$ & 0.79 & 0.184 & 0.245 & $\mathrm{GPa}$ \\
$H$ & 26.71 & 1.141 & 4.624 & \\
$C_{H}$ & 1.38 & 0.786 & 0.766 & $\mathrm{GPa}$ \\
$T_{M_{0}}$ & 1357 & 933.5 & 2380 & $\mathrm{~K}$ \\
$N_{0}$ & $10^{9}$ & & & $\mathrm{~cm}^{-2}$ \\
$M$ & $10^{11}$ & & & $\mathrm{~cm}^{-2}$ \\
$n$ & & $\infty$ & $\infty$ & \\
$n_{1}$ & 0.49 & 0.151 & 0.8 & \\
$m$ & 1.00 & 1.00 & 1.00 & \\
$\tau_{0}$ & $8.5 \times 10^{-5}$ & & & $\mu \mathrm{s} \mathrm{cm}^{-2}$ \\
$\tau_{0}^{\prime}$ & & 1.00 & 1.00 & $\mathrm{~s}$ \\
\hline \hline
\end{tabular}


equations. The algorithm works in a fixed Cartesian grid with state variables stored at cell centers. Cell wall fluxes are reconstructed using a third-order weighted essentially nonoscillatory scheme [26]. A third-order stability-preserving Runge-Kutta method [26] is employed for time stepping. The plastic update can be reduced to a stiff ordinary differential equation dependent only on the local stress state. For the particular equation of state (6), an exact solution for this ordinary differential equation can be obtained. The free surface between solid and vacuum is modeled using a level-set boundary-capturing algorithm combined with the modified ghost fluid method [27]. This technique allows us to track the interface position and impose the correct boundary conditions in the solid material without having to populate the entire vacuum region with a fictional low-density material. Indeed, no time evolution of the equations of motion in the vacuum region needs to be performed.

\section{ESTIMATION OF THE $C$ COEFFICIENT}

We now describe the process for obtaining a value for the coefficient $C$ in the expression

$$
\bar{\eta}_{s p}=C \frac{\rho_{0} \dot{\eta}_{s p, \max }^{2}}{k \sigma_{Y}},
$$

based on numerical simulations using our multimaterial Eulerian solver for elastic-plastic solids. The computational domain employed is rectangular with vacuum on the left and a solid material on the right. A shock is produced in the solid by means of setting up a Riemann problem at the initial computation time, which results in a shock wave moving to the left in the $x$ direction towards the interface and a secondary shock moving towards the computational domain boundary, which has an outflow boundary condition. The interface is colocated perpendicularly to the propagation direction of the shock and is perturbed by a sinusoidal wave. The relationships between the length and width of the computational domain and the wave number of the interface perturbation are $k \Delta x=6 \pi$ and $k \Delta y=2 \pi$. The change in mean velocity of the interface after the shock interaction is estimated and added to the initial velocity of all the regions, such that when the instability of the interface develops, its mean position remains approximately stationary.

A perfectly plastic model is always used in these simulations, as the yield stress must be known in order to obtain the coefficient $C$ through Eq. (10). In this case, the relaxation time in Eq. (8) is reduced to

$$
\tau=\tau_{0}\left(\frac{\sigma_{Y}}{\sigma_{m}}\right)^{n} \text { with } n \rightarrow \infty .
$$

For each of the materials considered, the initial amplitude of the interface relative to the wave number $\eta_{0} k$, the jump in velocity behind the shock (i.e., the strength of the shock) $\Delta u_{s}$, and the yield stress $\sigma_{Y}$ are varied according to the values in Table III. For those simulations that result in a stable long-term behavior (there are cases in which the combination of initial conditions leads to an unstable nonlinear behavior of the interface), the values of the mean amplitude in the long term and the maximum growth rates of spikes and bubbles are computed. The amplitudes of spikes and bubbles
TABLE III. Parametric study of the Richmyer-Meshkov flow for solid-vacuum interfaces in planar geometry.

\begin{tabular}{lcc}
\hline \hline Materials & & $\begin{array}{c}\text { OFHC copper, } \\
\text { aluminum Al6061-T6, } \\
\text { stainless steel SS 304 }\end{array}$ \\
\hline Initial amplitude parameter & $\eta_{0} k$ & $0.125,0.18,0.22,0.4$ \\
Jump in velocity across shock & $\Delta u_{s}$ & $1.25,2.5 \mathrm{~km} / \mathrm{s}$ \\
Yield stress & $\sigma_{Y}$ & $0.25,0.5,0.75,1.0 \mathrm{GPa}$ \\
\hline \hline
\end{tabular}

are computed by comparison with the time evolution of the amplitude position in a simulation set up with the same initial parameters except for $\eta_{0} k=0$. Figure 2 shows a dot for the result of each simulation and a comparison to the previous work of Dimonte et al. [14]. The result of the linear regression is also depicted. For the bubble amplitudes, we additionally compare our results with those predicted by the analytical model of Mikaelian [28]

$$
\eta k=\eta_{0} k+\frac{2}{3} \ln \left(1+\frac{\rho_{0} U_{b u}^{2}}{2 \sigma_{Y}}\right),
$$

where $U_{b u}$ is the bubble maximum growth rate. This expression derives from the growth rate of the Richmyer-Meshkov instability predicted for viscous fluids and the analogy that can be drawn between the viscous stress and the yield stress of solid materials.

The coefficient for the linear fit that is obtained from our simulations is $C \approx 0.22$ while Dimonte et al. computed a value $C \approx 0.24$. This difference in the slope of the linear regression may look large at first but a closer examination of the data points reveals that for small initial amplitudes, i.e., results

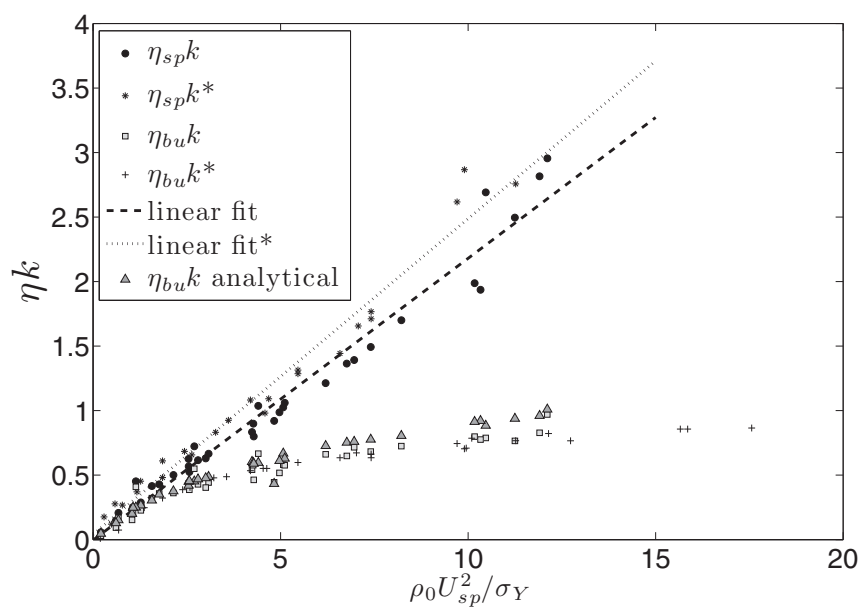

FIG. 2. Maximum nondimensional spike amplitude $\left(k \eta_{s p}\right)$ vs maximum nondimensional spike growth rate squared $\left(\rho_{0} U_{s p}^{2} / \sigma_{Y}\right)$ for different initial conditions, shock strengths, and yield stresses. Circles represent AMROC spike results. Dimonte et al. results [14] for spikes are denoted by stars. The dashed and dotted lines are the linear regressions obtained from the data points for AMROC and PAGOSA simulations, respectively. Finally, maximum bubble amplitudes are also shown using squares, crosses, and triangles for AMROC simulations, PAGOSA simulations [14], and Mikaelian's theoretical solution (12), respectively. 


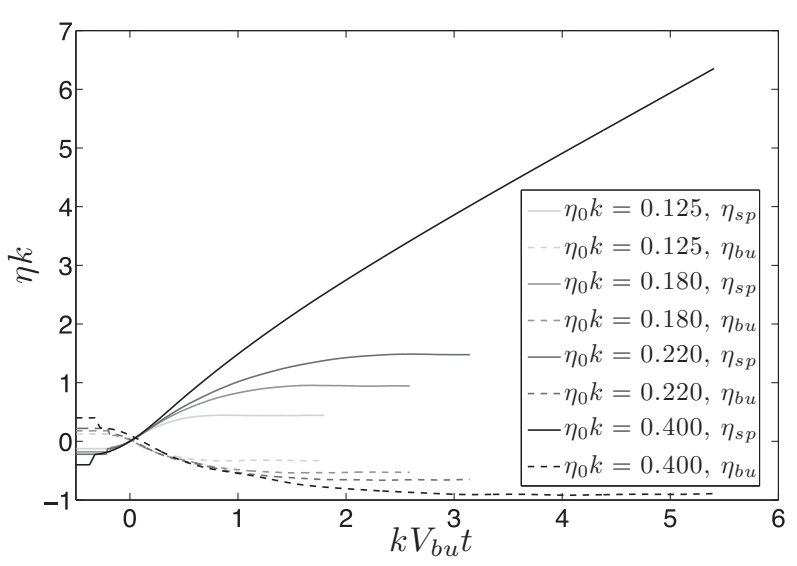

(a)

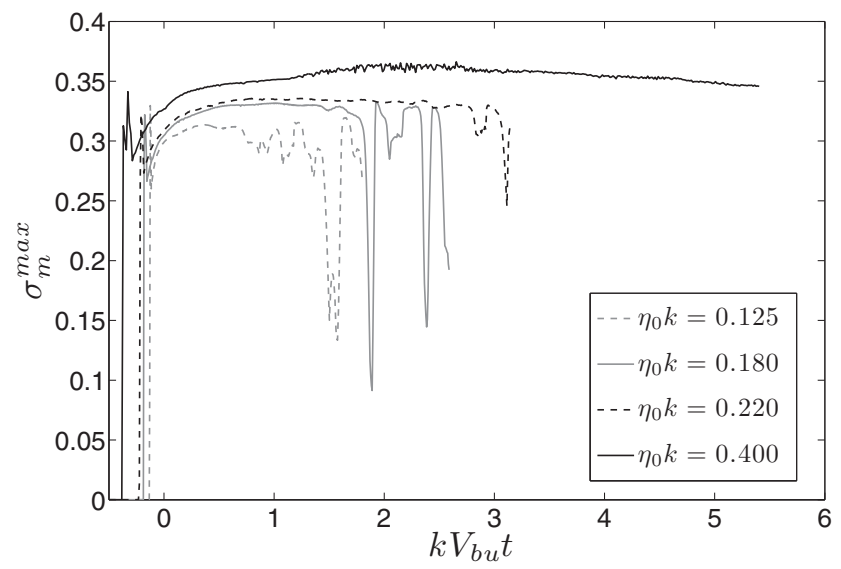

(b)

FIG. 3. Evolution in time of (a) spike and bubble amplitudes ( $\eta_{s p}$ and $\eta_{b u}$, respectively) and (b) maximum von Mises stress $\sigma_{m}^{\max }$ for OFHC copper.

closer to a linear regime, the values obtained are very similar in both approaches. It is only as the saturation amplitude grows that the obtained results exhibit an underestimation of the linear fit while those of Dimonte et al. are consistently above the linear regression function. If only data points with $k \eta_{s p}^{\max }<2$ are considered, the gap in slopes of the linear fits becomes noticeably smaller. Additionally we note that the data points that correspond to higher initial amplitude values are consistently below the linear fit line than those values obtained employing the same material, yield stress, and shock strength but lower initial amplitude. This phenomenon is most likely a consequence of the phase reversal that occurs shortly after the shock processes the interface and suggests that Eq. (10) may need to be modified to

$$
\bar{\eta}_{s p}-\eta_{s p}^{\prime 0}=C \frac{\rho_{0} \dot{\eta}_{s p, \max }^{2}}{k \sigma_{Y}},
$$

where $\eta_{s p}^{\prime 0}$ is some factor of the initial amplitude. However, attempts at setting $\eta_{s p}^{\prime 0}$ to the initial amplitude or the amplitude immediately after the shock-interface interaction have been

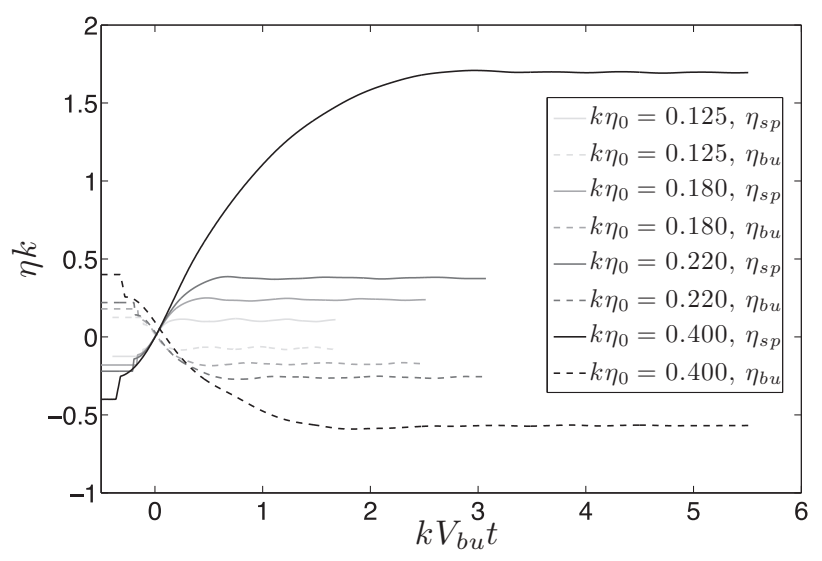

(a) unsuccessful in correcting the results. The coefficients of the linear regression obtained when only results for each of the materials are considered are very similar to the value of $C \approx$ 0.22 . With respect to the bubble saturation amplitudes, our results closely match those produced by the PAGOSA code and the analytical expression (12).

\section{SIMULATIONS WITH CALIBRATED PLASTICITY MODELS}

The linear regression law obtained in the last section is used here to infer an effective yield stress when the plasticity behavior of the material is not perfectly plastic. For each of the materials, cases with initial amplitudes $\eta_{0} k=0.125,0.18,0.22$, and 0.4 are considered with $\Delta u_{s}=$ $1.25 \mathrm{~km} / \mathrm{s}$. The choice of a relatively weak shock is motivated to avoid reaching temperatures that would induce melting. When melting occurs, the effective yield stress reduces to zero and we recover the typical unstable behavior of a fluid. Computing the long-term amplitude of the spikes and the maximum growth rate, the effective yield stress that determines

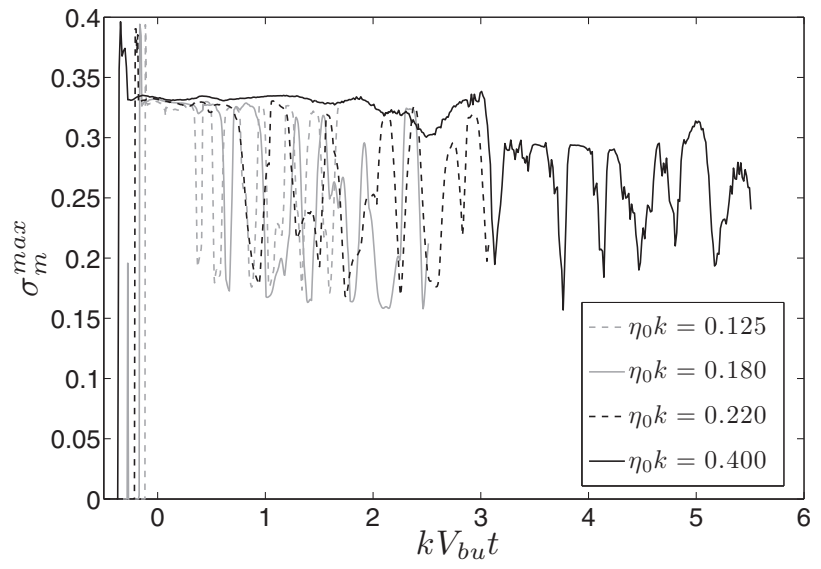

(b)

FIG. 4. Evolution in time of (a) spike and bubble amplitudes ( $\eta_{s p}$ and $\eta_{b u}$, respectively) and (b) maximum von Mises stress $\sigma_{m}^{\max }$ for aluminum Al6061-T6. 


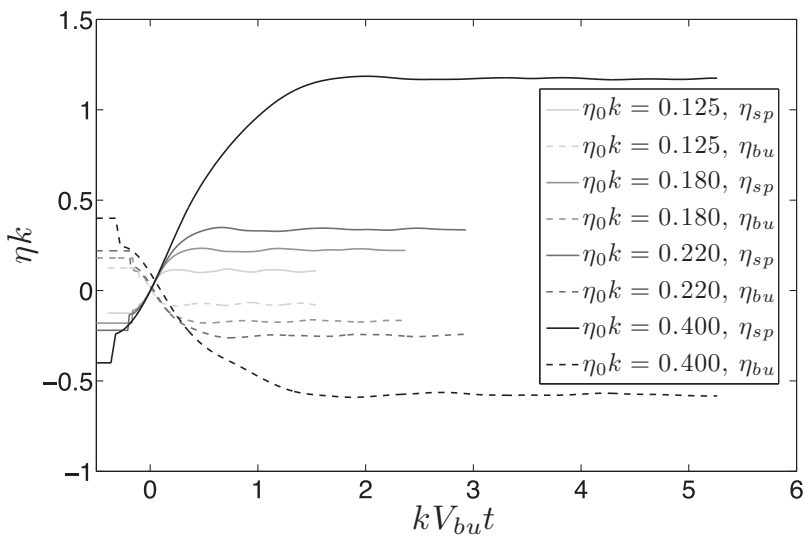

(a)

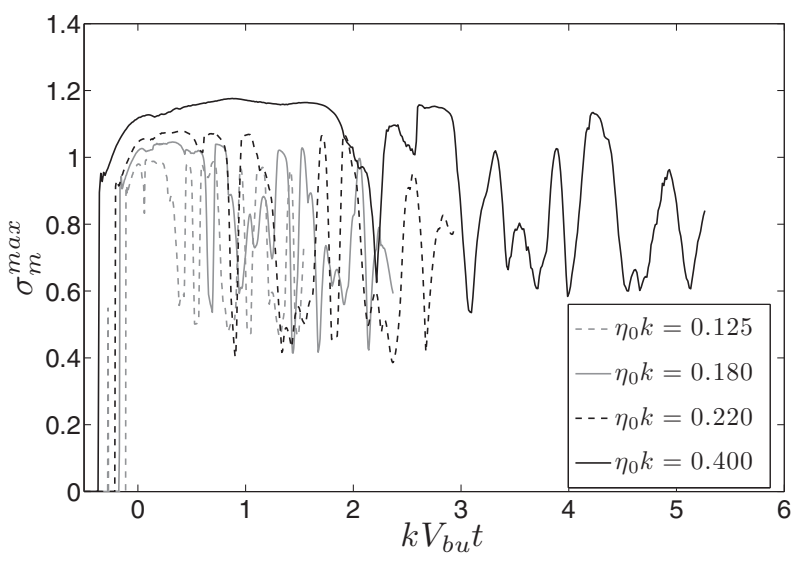

(b)

FIG. 5. Evolution in time of (a) spike and bubble amplitudes ( $\eta_{s p}$ and $\eta_{b u}$, respectively) and (b) maximum von Mises stress $\sigma_{m}^{\max }$ for stainless steel SS-304.

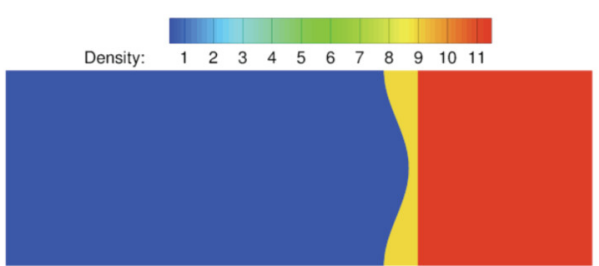

(a)Initial condition

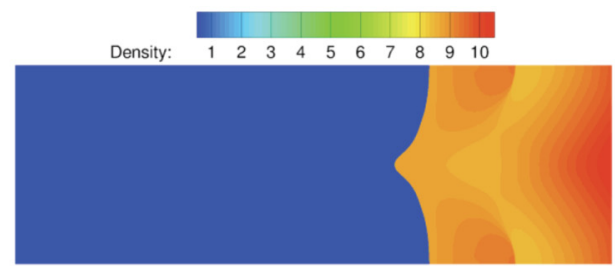

(c) $t V_{b u} k=0.56$

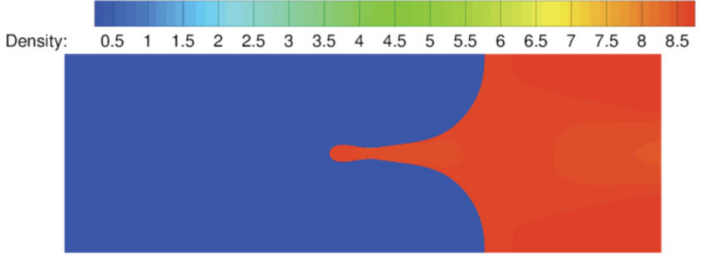

(e) $t V_{b u} k=2.88$

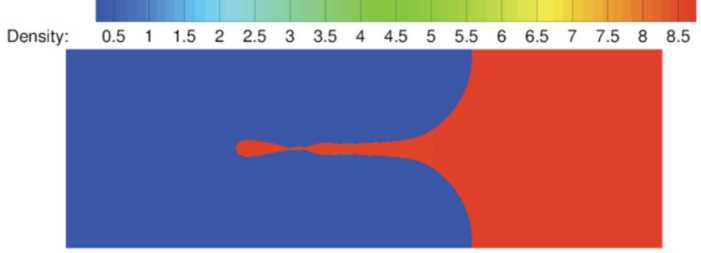

(g)t $V_{b u} k=5.54$

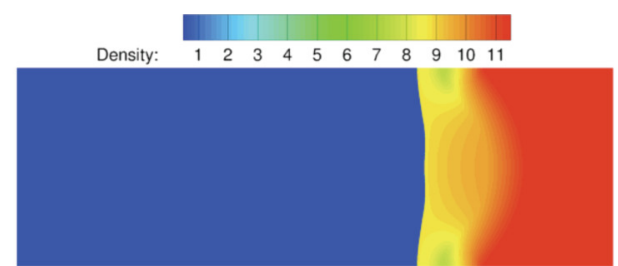

(b) $t V_{b u} k=0.01$

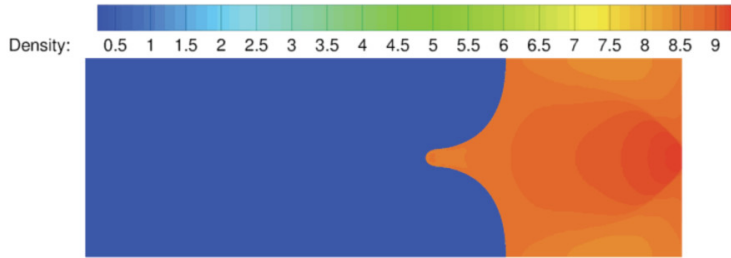

(d) $t V_{b u} k=1.33$

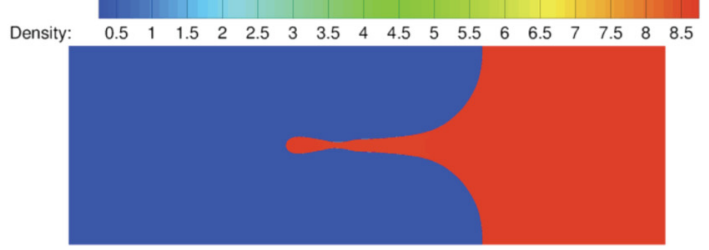

(f) $t V_{b u} k=4.10$

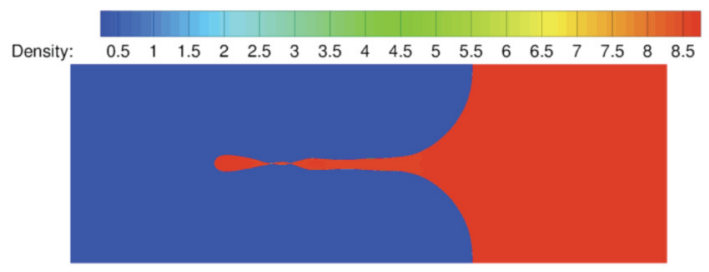

(h) $t V_{b u} k=6.32$

FIG. 6. (Color online) Sequence of density contour plots that describe the evolution of the Richtmyer-Meshkov instability of a coppervacuum interface. The calibrated plasticity model for copper is employed with $\eta_{0} k=0.4$ and $\Delta u_{s}=1.25 \mathrm{~km} / \mathrm{s}$. The interface exhibits unstable behavior that results in the formation of ejecta. 
TABLE IV. Comparison of effective yield stress. $\sigma_{\text {eff }}$ and $\sigma_{m}$ are computed from Eq. (14) and by inspection of Figs. 3(b), 4(b), and 5(b), respectively. N/A indicates not available since the interface amplitude constantly grows in this particular case.

\begin{tabular}{|c|c|c|c|c|c|c|c|c|c|c|c|c|}
\hline \multirow[b]{2}{*}{$\eta_{0} k$} & \multicolumn{4}{|c|}{ OFHC copper } & \multicolumn{4}{|c|}{ Aluminum Al6061-T6 } & \multicolumn{4}{|c|}{ Stainless steel SS-304 } \\
\hline & 0.125 & 0.180 & 0.220 & 0.400 & 0.125 & 0.180 & 0.220 & 0.400 & 0.125 & 0.180 & 0.220 & 0.400 \\
\hline$\sigma_{\text {eff }}$ & 0.332 & 0.363 & 0.382 & N/A & 0.338 & 0.350 & 0.371 & 0.376 & 0.887 & 1.006 & 1.075 & 1.321 \\
\hline$\sigma_{m}$ & 0.313 & 0.335 & 0.335 & N/A & 0.327 & 0.332 & 0.334 & 0.335 & 0.983 & 1.037 & 1.077 & 1.176 \\
\hline
\end{tabular}

the transition from an unstable to a stable material interface is

$$
\sigma_{\mathrm{eff}}=0.22 \frac{\rho_{0} \dot{\eta}_{s p, \max }^{2}}{\bar{\eta}_{s p} k} .
$$

Figures 3-5 depict, for copper, aluminum, and stainless steel, respectively, the time evolution of the amplitudes of spikes and bubbles and the maximum von Mises stress attained at the interface. The time has been set to zero in the moment of the phase reversal [i.e., $\eta_{s p}(0)=0$ ]. When the calibrated plasticity models are used, the spike and bubble amplitudes follow the behavior first described in [13], exhibiting an initial linear growth of the interface followed by the stabilization around a long-term average amplitude. In the case of copper with $\eta_{0} k=0.4$, the interface enters a nonlinear regime before relaxation of stresses occurs and a purely unstable behavior of

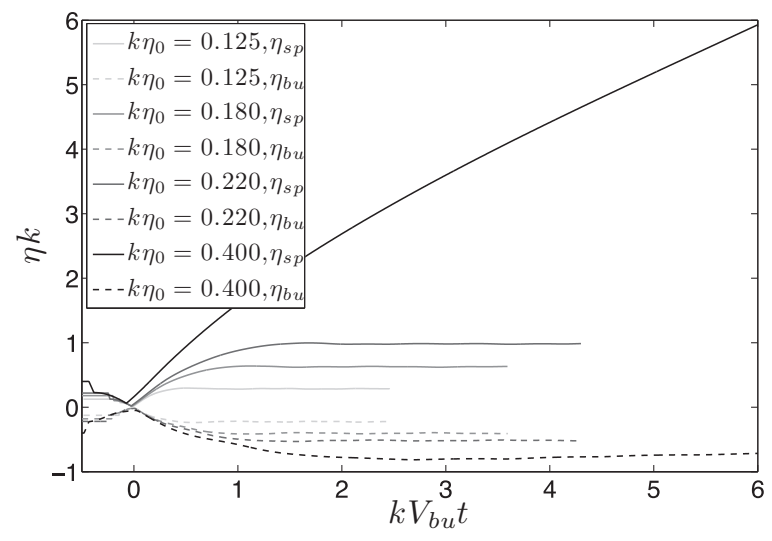

(a) $\sigma_{Y}=0.5 \mathrm{GPa}, \Delta u_{s}=1.25 \mathrm{~km} / \mathrm{s}$

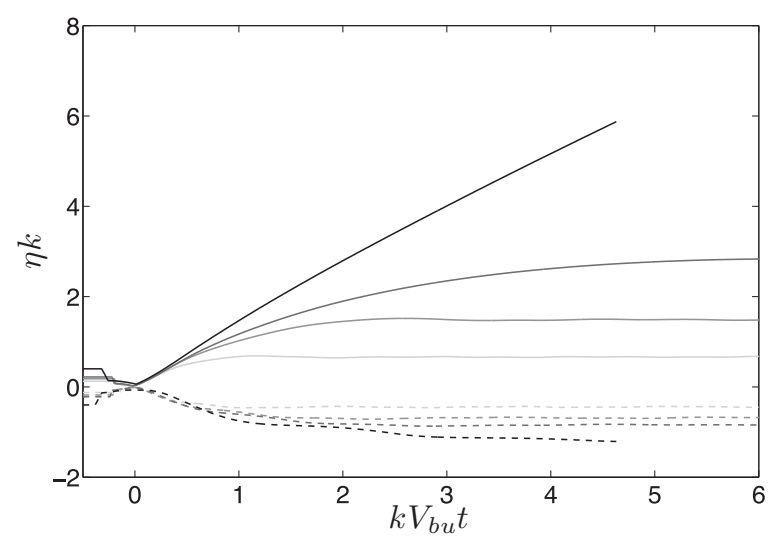

(c) $\sigma_{Y}=0.75 \mathrm{GPa}, \Delta u_{s}=2.5 \mathrm{~km} / \mathrm{s}$ the interface follows. After the spikes get extremely narrow, the tip of the spike detaches from the main material, forming ejecta (see Fig. 6). Regions of high von Mises stress commonly correspond to the central regions of spikes and bubbles, as the largest deformations occur there. The results show that, just after the shock-interface interaction, the von Mises stresses increase and reach the effective yield stress value sought here. As relaxation of stresses occurs and the interface transitions to a stable behavior, the material is no longer in a plastic state and the stress state describes an oscillatory behavior that follows the oscillations of the interface around the long-term mean value. Table IV compares the effective yield stress computed from Eq. (14) and that obtained from direct inspection of Figs. 3(b), 4(b), and 5(b). The results agree relatively well, with errors not commonly exceeding $10 \%$ of the measured value.

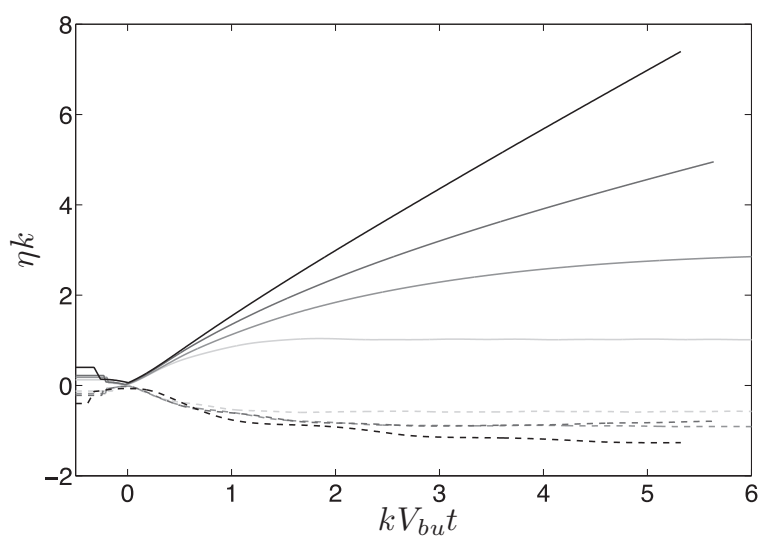

(b) $\sigma_{Y}=0.5 \mathrm{GPa}, \Delta u_{s}=2.5 \mathrm{~km} / \mathrm{s}$

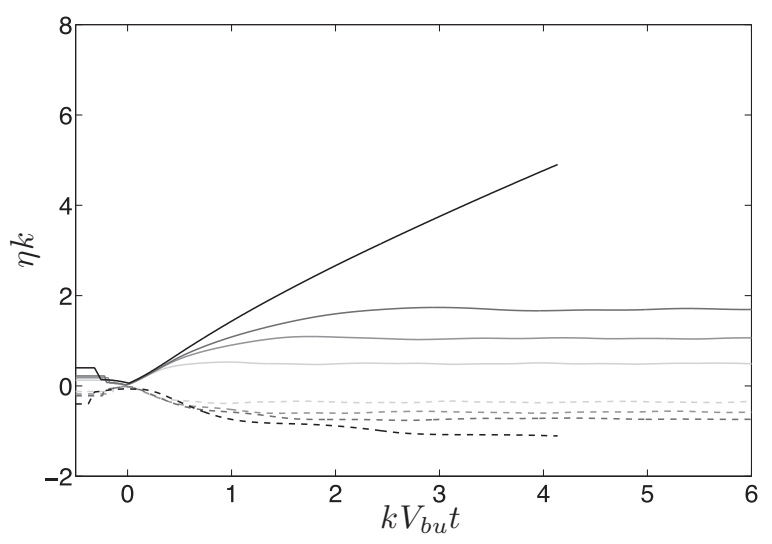

(d) $\sigma_{Y}=1.0 \mathrm{GPa}, \Delta u_{s}=2.5 \mathrm{~km} / \mathrm{s}$

FIG. 7. Evolution in time of spike and bubble amplitudes ( $\eta_{s p}$ and $\eta_{b u}$, respectively) for perfectly plastic copper under different yield stress values and shock strength conditions. 
The transition from saturation of the interface amplitude to unbounded growth appears to depend not only on the initial amplitude but also on material parameters (yield stress, shear strength, density) and the strength of the shock. Among the cases shown in Figs. 3-5, only copper with $\eta_{0} k=0.4$ exhibits unbounded growth. We can argue that the difference in behavior between materials is related to the nondimensional ratio between the yield, the density, the growth rate, and the yield stress $\rho_{0} \dot{\eta}_{s p \text {, max }}^{2} / \sigma_{Y}$. This relation is higher in copper than in stainless steel and aluminum, leading to higher spike amplitudes at equal shock conditions per Eq. (10). This seems to indicate that unbounded growth is related to situations in which the spikes, still in the plastic regime, reach a critical amplitude $(\eta k)_{c}$ beyond which the theory [13], which predicts interface restraint in all cases, does not apply. In [14], PAGOSA simulations for copper modeled as a perfectly plastic material with $\sigma_{Y}=0.5 \mathrm{GPa}$ and excited by a shock wave with $\Delta u_{s}=2.5 \mathrm{~km} / \mathrm{s}$ (twice as high as our value) are reported and results show that initial amplitudes $\eta_{0} k=0.22$ and 0.4 produce unbounded growth [these results can be compared to Fig. 7(b)]. In the same article, simulations run with the molecular dynamics code SPASM produce results with saturation of the interface for $\eta_{0} k=1$ and $\Delta u_{s}=1.5 \mathrm{~km} / \mathrm{s}$ (similar to the shock strength used in our simulations) but the calculated yield strength of the material is $\sigma_{Y}=1.1 \mathrm{GPa}$ compared to the value of $\approx 0.38 \mathrm{GPa}$ given by our calibrated plasticity model. Experimental results by Buttler et al. [29] reveal the existence of unbounded growth for high initial perturbation amplitudes. However, due to the small shock strengths used compared to our simulations, the cases $\eta_{0} k=0.12$ and 0.35 for copper exhibit a restrained free surface. To complement the evidence found in the literature that the material and shock strength have an influence on the unbounded growth of the free surface, we depict in Fig. 7 the evolution of spike and bubble amplitudes for perfectly plastic copper with different yield stresses $\left(\sigma_{Y}=0.5,0.75\right.$, and $\left.1.0 \mathrm{GPa}\right)$ and shock strengths $\left(\Delta u_{s}=1.25\right.$ and $\left.2.5 \mathrm{~km} / \mathrm{s}\right)$. Results suggest that the initial amplitude required to obtain ejecta increases as the yield stress increases and the shock strength decreases. However, the formulation of a criterion for the transition to unbounded growth may require a more extensive and detailed parametric study.

\section{CONCLUSION}

This paper extended the work in [13,14] to multiple materials with plasticity laws calibrated by experimental evidence. The linear expression that relates the saturation amplitude of the spikes with the yield stress, maximum growth rate, and density of the material was obtained for three different materials with distinct shear stress, bulk modulus, and thermal behavior, proving that this law is universal and relies exclusively on the yield stress and density of the material. This expression seems to apply even when large perturbation amplitudes that exceed the validity of the small-perturbation theory upon which this relation was developed in [13] are obtained. Even when results from cases with large initial amplitudes yield points slightly below the linear fit slope, attempts to improve the law by modifying some of the parameters involved in it to better represent the nonlinear regime have been unsuccessful. An effective yield stress was computed using the linear regression with coefficient $C=0.22$ for materials whose yield stress changes with hardening, strain rate, and thermal softening. Comparison with the von Mises stress obtained while the region of the material close to the interface is in a plastic state reveals that this effective yield stress, which was computed uniquely from the values of the long-term spike amplitude and the maximum growth rate, matches fairly well the real value of the yield stress before the material leaves the plastic state. Finally, there exist cases in which the spikes are not restrained by stress relaxation. The derivation of an exact criterion to separate unbounded growth to amplitude saturation requires an extensive parametric study considering variations in initial amplitude, material strength, and shock strength.

\section{ACKNOWLEDGMENT}

This material is based upon work supported by the Department of Energy National Nuclear Security Administration under Award No. DE-FC52-08NA28613.
[1] Robert D. Richtmyer, Commun. Pure Appl. Math. 13, 297 (1960).

[2] E. E. Meshkov, NASA Tech. Transcriptions F-13, 074 (1970).

[3] Gregory R. Baker, Daniel I. Meiron, and Steven A. Orszag, J. Fluid Mech. 123, 477 (1982).

[4] V. Wheatley, D. I. Pullin, and R. Samtaney, Phys. Rev. Lett. 95, 125002 (2005)

[5] John D. Lindl, Robert L. McCrory, and E. Michael Campbell, Phys. Today 45(9), 32 (1992).

[6] David Arnett, Astrophys. J. 127, 213 (2000).

[7] B. Fryxell, D. Arnett, and E. Mueller, Astrophys. J. 367, 619 (1991).

[8] Stephen P. Maran, George Sonneborn, Chun S. J. Pun, Peter Lundqvist, Rosina C. Iping, and Theodore R. Gull, Astrophys. J. 545, 390 (2000).
[9] J. M. Stone, J. Xu, and L. G. Mundy, Nature (London) 377, 315 (1995).

[10] A. R. Piriz, J. J. López Cela, N. A. Tahir, and D. H. H. Hoffmann, Phys. Rev. E 74, 037301 (2006).

[11] Jeeyeon N. Plohr and Bradley J. Plohr, J. Fluid Mech. 537, 55 (2005).

[12] A. López Ortega, D. J. Hill, D. I. Pullin, and D. I. Meiron, Phys. Rev. E 81, 066305 (2010).

[13] A. R. Piriz, J. J. López Cela, N. A. Tahir, and D. H. H. Hoffmann, Phys. Rev. E 78, 056401 (2008).

[14] Guy Dimonte, G. Terrones, F. J. Cherne, T. C. Germann, V. Dupont, K. Kadau, W. T. Buttler, D. M. Oro, C. Morris, and D. L. Preston, Phys. Rev. Lett. 107, 264502 (2011).

[15] W. N. Weseloh, S. P. Clancy, and J. W. Painter, Los Alamos National Laboratory, Technical Report No. LAUR-14425-M, 2010 (unpublished). 
[16] J. Marsden and T. Hughes, Mathematical Foundations of Elasticity (Dover Publications, New York, 1993).

[17] V. N. Dorovskii, A. M. Iskol'dskii, and E. I. Romenskii, J. Appl. Mech. Tech. Phys. 24, 454 (1983).

[18] V. A. Titarev, E. Romenski, and E. F. Toro, Int. J. Numer. Methods Eng. 73, 897 (2008).

[19] G. Ravichandran (private communication).

[20] W. G. Guo, Baozha Yu Chongji/Explosion and Shock Waves 25, 244 (2005) (in Chinese).

[21] Alejandro Lopez Ortega, Ph.D. thesis, California Institute of Technology, 2013.

[22] A. López Ortega, M. Lombardini, D. I. Pullin, and D. I. Meiron, J. Comput. Phys. 257, 414 (2014).

[23] P. T. Barton, R. Deiterding, D. Meiron, and D. Pullin, J. Comput. Phys. 240, 76 (2013).
[24] R. Deiterding, in Adaptive Mesh Refinement-Theory and Applications, edited by T. Plewa, T. Linde, and V. G. Weirs, Lecture Notes in Computational Science and Engineering (Springer, Berlin, 2005), pp. 361-372.

[25] M. J. Berger and P. Colella, J. Comput. Phys. 82, 64 (1989).

[26] Guang-Shan Jiang and Chi-Wang Shu, J. Comput. Phys. 126, 202 (1996).

[27] T. G. Liu, B. C. Khoo, and K. S. Yeo, J. Comput. Phys. 190, 651 (2003).

[28] Karnig O. Mikaelian, Phys. Rev. E 87, 031003 (2013).

[29] W. T. Buttler, D. M. Or, D. L. Preston, K. O. Mikaelian, F. J. Cherne, R. S. Hixson, F. G. Mariam, C. Morris, J. B. Stone, G. Terrones, and D. Tupa, J. Fluid Mech. 703, 60 (2012). 